\title{
Lumbar Spinal Canal Stenosis Classification Criteria: A New Tool
}

\author{
Parisa Azimi ${ }^{1}$, Hassan Reza Mohammadi ${ }^{1}$, Edward C. Benzel ${ }^{2}$, Sohrab Shahzadi ${ }^{1}$, Shirzad Azhari ${ }^{1}$ \\ ${ }^{1}$ Department of Neurosurgery, Shahid Beheshti University of Medical Sciences, Tehran, Iran \\ ${ }^{2}$ Department of Neurosurgery, Cleveland Clinic Foundation, Cleveland, OH, USA
}

Study Design: Case-control study.

Purpose: To design a new tool for classifying lumbar spinal canal stenosis (CLSCS).

Overview of Literature: Grading of patients with lumbar spinal canal stenosis (LSCS) is controversial.

Methods: The Oswestry disability index (ODI) and the neurogenic claudication outcome score (NCOS) were recorded. Four parameters, which indicate the severity of LSCS disease, including Hufschmidt-grade, grading of magnetic resonance imaging, self-paced walking test, and stenosis ratio (SR) were employed. For the SR, quartile analysis was applied for classifying LSCS and the Hufschmidt-grade was modified into a 4-grade score. An initial score was assigned to each metric based on the severity of LSCS. Using the inverse-variance weighting method, the relative weights of these domains and their categories were determined. The score for all of the cases was obtained based on their weight by summing up the points of the four variables. Quartile analysis was used and a CLSCS score was proposed. Finally, intra- and interobserver reliability, and validity were assessed.

Results: A total of 357 patients were studied. The final CLSCS score for each case ranged from 4 to 16.5. Based on the quartile analysis, using the new criteria set, the CLSCS score was divided into four categories: CLSCS $<7$ (grade 0); 7 $\leq L S C S<10$ (grade 1); $10 \leq$ CLSCS $<13$ (grade 2); and 13 $\leq$ CLSCS $\leq 16.5$ (grade 3). The kappa values of for the CLSCS score indicated a perfect agreement. The CLSCS was correlated with the ODI and NCOS. All patients with grade 3 CLSCS were observed in the surgical group.

Conclusions: The CLSCS score can be helpful for classifying LSCS patients and in the decision-making process.

Keywords: Lumbar spinal canal stenosis; New tool; Classification; Classifying lumbar spinal canal stenosis

\section{Introduction}

A common cause of low back pain and disability associated with neural element compression is lumbar spinal canal stenosis (LSCS). LSCS is a condition in which the degenerative central canal narrows due to degenerative disc bulging, hypertrophied ligamentum flavum, and degenerative changes in the facet joint [1]. The symptoms of LSCS include pain in the groin, hips, and buttocks, and numbness or weakness in the legs and lower back. Symptoms usually worsen with walking or standing (claudication) [1]. The ability to classify LSCS patients is important for choosing the appropriate management. A wide range of clinical, electrodiagnostic, and radiological tests are currently used to define and classify LSCS. However, there is no generally accepted "gold standard" for the classification of LSCS and for the decision-making process in clinical practice, especially in borderline patients [2,3]. Hence,

Received Nov 5, 2014; Revised Nov 25, 2014; Accepted Nov 25, 2014

Corresponding author: Parisa Azimi

Functional Neurosurgery Research Center of Shohada Tajrish Hospital, Shahid Beheshti University of Medical Sciences,

Sharadari St., Tajrish Square, Tehran 1989934148, Iran

Tel: +98-2122749204, Fax: +98-2188265188, E-mail: parisa.azimi@gmail.com 
there is a need for consensus on the criteria for classifying LSCS so as to provide a decision-making tool that helps in deciding whether surgical or conservative management should be performed in LSCS. This study sought to develop a new quantitative tool based on the Hufschmidtgrade, the grading of magnetic resonance imaging (MRI), the self-paced walking test (SPWT), and the stenosis ratio (SR) to classify LSCS patients.

\section{Materials and Methods}

\section{Patients and data collection}

Cases of LSCS diagnosed at a single center were identified for this retrospective study, between February 2007 and April 2012, in a large teaching hospital in Tehran, Iran. The diagnosis of LSCS was established based on the clinical symptoms, neurological examination, and imaging studies-including plain radiography, MRI, and computed tomography (CT) of the lumbar spine. All of the patients had typical symptoms of LSCS, such as neurogenic intermittent claudication and leg pain and/or numbness. In all of the patients, the diagnosis was confirmed by more than one spine surgeon. The level(s) of stenosis were explored on the MRI or CT. There were no restrictions on patient choice with regard to level(s) of LSCS, age, or other characteristics. The exclusion criteria were prior lumbar spine surgery and congenital spine anomalies.

Demographics, including age, gender, and body weight, were recorded. The duration of symptoms (in months) and neurogenic claudication outcome score (NCOS) were recorded. The NCOS ranges from 0 to 100 . Higher scores have been associated with a better clinical outcome.

\section{Additional metrics}

\section{1) The $S R$}

The SR is defined as the ratio of the cross-sectional area of the spinal canal on the axial MRI image at the disc level that is associated with greatest neurologic compression to the cross-sectional area at the next rostral pedicle level above [4]. It has been used to determine the severity of stenosis. The cross-sectional area was measured by the Hamanishi technique [5]. The calculations were performed by two independent surgeons and they were blinded to each other's results. The SRs were divided into quartiles of severity of lumbar stenosis.

\section{2) The SPWT}

The SPWT is a measure of walking capacity, which is the distance a person with LSCS is able to walk without support on a level surface at a self-selected speed before being forced to stop due to symptoms of LSCS [6]. The SPWT is presented as a feasible and reproducible criterion measure for use in LSCS and NC patients $[7,8]$. The SPWT was extracted from patients' case records and categorized as poor (less than $100 \mathrm{~m}$ ), fair (between $100 \mathrm{~m}$ and $800 \mathrm{~m}$ ), good (between $800 \mathrm{~m}$ and 1,600 m), and very good (more than $1,600 \mathrm{~m})$ [9].

\section{3) The grading of MRI}

Lee et al. [10] described a 4-grade (0, 1, 2, and 3) classification of the severity of LSCS based on the degree of separation of the cauda equina on T2-weighted axial images, without measurement of the parameters. They defined grade 0 , no lumbar stenosis without obliteration of the anterior CSF space; grade 1, mild stenosis with separation of all cauda equina; grade 2 , moderate stenosis with some cauda equina aggregated making it impossible to visually separate them; and grade 3 , severe stenosis with none of the cauda equina separated.

\section{4) Hufschmidt-grade}

Grading of LSCS presented by Hufschmidt and Bar [11] was categorized as follows: grade I; neurogenic intermittent claudication characterized by a reduced walking distance (caused by pain) and short term intermittent sensory and/or motor deficits that at rest might be unremarkable, but might worsen while walking, grade II; intermittent paresis refers to already persistent sensory deficits, loss of reflexes and intermittent paresis, and grade III; persistent, progressive paresis, accompanied by partial regression of pain. Grade I was broken down into grade 0 (symptoms after walking $100 \mathrm{~m}$ or more than $100 \mathrm{~m}$ ) and grade 1 (symptoms after walking less than $100 \mathrm{~m}$ ). The Hufschmidt-grade was, henceforth, modified from a 3- to a 4-grade scale.

\section{5) The Iranian version of the ODI}

This is a measure of functionality and contains 10 items. The possible score on the Oswestry disability index (ODI), ranges from 0 to 50, with higher scores indicating worse conditions. The psychometric properties of the Iranian version of the questionnaire are well documented [12].

Both subjective and objective parameters such as the 
Hufschmidt-grade, the grading of MRI, the SPWT, and the SR, were used to build the new quantitative tool.

\section{Treatment}

All of the patients had received surgery or conservative treatment for LSCS. All patients in the surgical group failed at least 6 months of conservative treatment, including oral analgesics, physiotherapy, and epidural steroids.

\section{Statistical analysis}

All statistical analyses were performed using the PASW ver. 18 (SPSS Inc., Chicago, IL, USA). The SR was grouped according to the severity of LSCS by performing quartile analysis with the SR $0.75<$ between $\leq 1$ (lowest quartile), $0.50<$ between $\leq 0.75$ (lower quartile), $0.25<$ between $\leq 0.50$ (higher quartile), and $0<$ between $\leq 0.25$ (highest quartile). For each of the four metrics (SR, Hufschmidt-grade, SPWT, and grading of MRI), an initial score of 1, 2, 3, and 4 was assigned to each metric based on the severity of LSCS [9-11]. Then, for each parameter, we added together all patients' scores and calculated the mean score and the corresponding standard deviation. Consequently, the variance for each parameter was calculated. Finally, using the inverse-variance weighting method (weight $=1$ / $\sigma^{2}$; variance $=6$ ), the relative weights of these domains and their categories were determined. Inverse-variance weighting was selected as it is typically used in statistical meta-analysis to combine the results from independent measurements and it is an appropriate method for creating a score. The score for all of the cases was obtained based on their weight by summing up the points of the four variables. Finally, the quartile analysis was used and a new classification for LSCS was proposed. For instance, for the SR, we first assigned a score of 1, 2, 3, and 4 based on the severity of LSCS in each case. Then, we added these scores for the whole sample size $(n=357)$ to calculate the mean score and standard deviation. Later, the standard deviation was powered by 2 to estimate the variance. The weight for SR was derived from the above formula, and therefore, to calculate the weighted score for each case the initial score for each case multiplied it. The procedure was repeated for each parameter, and the addition of these weighted scores yielded the final score for each case ranging from 4 to 16.5 .

\section{Assessment of the intra- and interobserver reliability of the classification grading}

In order to validate the classification, 83 patients with all types of disorders were randomly selected. Two independent observers classified each case twice within a 2-week interval to measure the intra- and inter-observer differences. The weighted Kappa coefficient was calculated for each spine surgeon based on his first and second data report and comparison of kappa between spine surgeons. Kappa values ranged between 0 and 1 ; the greater the kappa value, the higher the concordance rate. Kappa value of 0 to 0.20 indicated slight agreement, 0.21 to 0.40 indicated fair agreement, 0.41 to 0.60 indicated moderate agreement, 0.61 to 0.80 indicated substantial perfect agreement, and $\geq 0.81$ was regarded as almost perfect agreement according to the interpretation by Landis and Koch [13].

\section{Convergent validity}

The correlation between the CLSCS and the ODI and NCOS was assessed using the Pearson's correlation coefficient and values of 0.40 or above were considered satisfactory $(r \geq 0.81-1.0$; excellent, $0.61-0.80$; very good, $0.41-0.60$; good, $0.21-0.40$; fair, and $0.0-0.20$, poor) [14]

\section{Ethics}

The research was approved by the Ethics Committee of Shahid-Beheshti University of Medical Sciences, Tehran, Iran.

\section{Results}

Demographics of the LSCS patients and their scores for the SR, Hufschmidt-grade, SPWT, grading of MRI, the ODI, and the NCOS are shown in Table 1. The SR was broken down to classify LSCS into quartiles: no lumbar stenosis $(0.75<$ between $\leq 1)$, mild stenosis $(0.50<$ between $\leq 0.75)$, moderate stenosis $(0.25<$ between $\leq 0.50)$, and severe stenosis $(0<$ between $\leq 0.25)$. The classification scheme for LSCS and the relative weights of these domains and their categories are shown in Table 2.

Based on the quartile analysis using the PASW statistics, the CLSCS score was divided into 4 categories: CLSCS $<7$ (grade $0=$ no lumbar stenosis); $7 \leq \mathrm{CLSCS}<10$ (grade $1=$ 
Table 1. Baseline demographic data in patients with lumbar spinal canal stenosis $(\mathrm{n}=357)$

\begin{tabular}{|c|c|}
\hline Characteristics & Value \\
\hline Age (yr) & $58.1 \pm 10.6$ \\
\hline Gender (male, [\%]) & 46.8 \\
\hline Body weight (kg) & $77.3 \pm 10.4$ \\
\hline \multicolumn{2}{|l|}{ Lumbar stenosis levels } \\
\hline One-level & $36(10.1)$ \\
\hline Two-level & $139(38.9)$ \\
\hline Three-level & $131(36.7)$ \\
\hline Four-level & $51(14.3)$ \\
\hline \multicolumn{2}{|l|}{ Symptoms } \\
\hline Duration of symptoms (mo) & $39.6 \pm 21.1$ \\
\hline$\left.\mathrm{OD}\right|^{\mathrm{a})}$ & $33.4 \pm 10.2$ \\
\hline NCOS score ${ }^{\text {b) }}$ & $29.2 \pm 11.1$ \\
\hline \multicolumn{2}{|l|}{ SPWT ${ }^{\mathrm{c})}$} \\
\hline Very good & $76(21.3)$ \\
\hline Good & $72(20.2)$ \\
\hline Fair & 94 (26.3) \\
\hline Poor & $115(32.2)$ \\
\hline \multicolumn{2}{|l|}{ Grading of $\mathrm{MRI}^{\mathrm{d}}$} \\
\hline Grade 0 & $85(23.8)$ \\
\hline Grade 1 & $103(28.9)$ \\
\hline Grade 2 & $127(35.6)$ \\
\hline Grade 3 & 42 (11.8) \\
\hline \multicolumn{2}{|l|}{$\mathrm{SR}^{\mathrm{e})}$ by quartiles } \\
\hline$>0.75 \& \leq 1$ & $77(21.6)$ \\
\hline$>0.50 \& \leq 0.75$ & $98(27.5)$ \\
\hline$>0.25 \& \leq 0.50$ & $121(33.9)$ \\
\hline$>0 \& \leq 0.25$ & $61(17.1)$ \\
\hline \multicolumn{2}{|l|}{ Hufschmidt-grade ${ }^{f)}$} \\
\hline Grade 0 & $86(24.1)$ \\
\hline Grade 1 & $124(34.7)$ \\
\hline Grade 2 & $113(31.7)$ \\
\hline Grade 3 & 34 (9.5) \\
\hline
\end{tabular}

Values are presented as median \pm standard deviation or number (\%).

a) The Oswestry disability index (ODI) scores ranges from 0 to 50, with higher scores indicating worse conditions; ${ }^{b}$ The neurogenic claudication outcome (NCOS) score ranges from 0 to 100, with higher scores indicating less severe symptoms; ${ }^{\text {') } T h e ~ s e l f-p a c e d ~ w a l k i n g ~ t e s t ~(S P W T) ~}$ is a measure for walking capacity, and categorized as poor (less than $100 \mathrm{~m}$ ), fair (between 100 and $800 \mathrm{~m}$ ), good (between $800 \mathrm{~m}$ and $1,600 \mathrm{~m}$ ), and very good (more than 1,600 m). ${ }^{\mathrm{d}}$ Grading of magnetic resonance imaging (MRI): grade 3, severe stenosis; grade 2, moderate stenosis; grade 1, mild stenosis; and grade 0, no lumbar stenosis; ${ }^{e}$ The stenosis ratio (SR) is the cross-sectional area of the canal at the axial MRI image with greatest neurologic compression at disc level over the cross-sectional area at the pedicle level above; fil Hufschmidtgrade: severity of lumbar spinal stenosis as observed based on clinical symptoms. mild lumbar stenosis); $10 \leq \mathrm{CLSCS}<13$ (grade $2=$ moderate lumbar stenosis); and $13 \leq \mathrm{CLSCS} \leq 16.5$ (grade $3=$ severe lumbar stenosis). The score index for CLSCS was obtained by summing up the points of the four variables that are shown in Table 3.

The Kappa statistic was calculated for the CLSCS score and its domain, which are shown in Table 4. The kappa value for the CLSCS score for the intra-observer result was 0.82 (range, 0.81-0.93) and that for the inter-observer result was 0.84 (range, 0.82-0.96), indicating substantial perfect agreement. In addition, the CLSCS was strongly correlated with the ODI and NCOS, lending support to its good convergent validity $(r=0.81, p<0.001 ; r=0.83$, $p<0.001$, respectively).

In all, 171 out of the 357 patients underwent surgery and the remaining 186 patients received conservative treatment at the one-year follow-up from diagnosis. Overall, there were 32 patients who dropped out of the study or who were lost to follow-up. All patients in the surgical group failed at least 6 months of conservative treatment, including oral analgesics, physiotherapy, and epidural steroids. Treatments received within one year based on the CLSCS grade are shown in Table 5. Most of the patients $(94.7 \%)$ who underwent surgery were identified as having grade- 2 and grade- 3 stenosis, while those who received conservative treatment were identified as having grade- 0 and grade- 1 stenosis $(97.8 \%)$. This difference was statistically significant $(p<0.001)$ (Table 5$)$. All patients with grade- 3 stenosis failed conservative treatment and were observed in the surgical group, and all patients with grade- 0 stenosis were observed in the conservative group.

\section{Discussion}

A new classification criterion is presented in this study for LSCS patients based on the four classification grading systems; the Hufschmidt-grade, the grading of MRI, the SPWT, and the SR. Our findings suggest that the CLSCS score is also generally reliable and valid for classifying these patients. Thus, the CLSCS score can be used in the decision-making process. Although the CLSCS score showed promise, the sample size was small, and therefore, the technique will need to be repeated in larger, multicenter datasets to convincingly show its classification power.

To date, no studies presenting a quantitative grading system for the classification of LSCS patients have been 
Table 2. Classified of lumbar stenosis, for SPWT, grading of MRI, SR, and Hufschmidt-grade included in the study and theirs relative weight (n=357)

\begin{tabular}{|c|c|c|c|c|}
\hline \multirow{2}{*}{ Variable } & \multirow{2}{*}{ Initial weight } & \multirow{2}{*}{ Variance (б) } & \multicolumn{2}{|c|}{ Relative weight ${ }^{a)}$} \\
\hline & & & Exact & Rounded to 0.5 \\
\hline SPWT ${ }^{\text {b) }}$ & & 1.286 & 0.604 & \\
\hline Very good & 1 & & 0.604 & 0.5 \\
\hline Good & 2 & & 1.208 & 1 \\
\hline Fair & 3 & & 1.812 & 2 \\
\hline Poor & 4 & & 2.416 & 2.5 \\
\hline Grading of $\left.M R\right|^{c l}$ & & 0.942 & 1.126 & \\
\hline Grade 0 & 1 & & 1.126 & 1 \\
\hline Grade 1 & 2 & & 2.252 & 2 \\
\hline Grade 2 & 3 & & 3.378 & 3.5 \\
\hline Grade 3 & 4 & & 4.504 & 4.5 \\
\hline$S R^{d)}$ by quartiles & & 1.025 & 0.952 & \\
\hline$>0.75 \& \leq 1$ & 1 & & 0.952 & 1 \\
\hline$>0.50 \& \leq 0.75$ & 2 & & 1.904 & 2 \\
\hline$>0.25 \& \leq 0.50$ & 3 & & 2.856 & 3 \\
\hline$>0 \& \leq 0.25$ & 4 & & 3.808 & 4 \\
\hline Hufschmidt-grade ${ }^{e)}$ & & 0.870 & 1.321 & \\
\hline Grade 0 & 1 & & 1.321 & 1.5 \\
\hline Grade 1 & 2 & & 2.642 & 2.5 \\
\hline Grade 2 & 3 & & 3.963 & 4 \\
\hline Grade 3 & 4 & & 5.284 & 5.5 \\
\hline
\end{tabular}

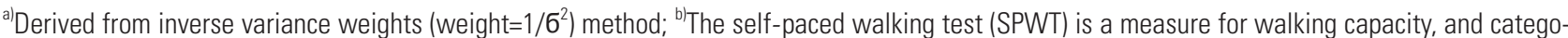
rized as poor (less than $100 \mathrm{~m}$ ), fair (between $100 \mathrm{~m}$ and $800 \mathrm{~m}$ ), good (between $800 \mathrm{~m}$ and 1,600 m), and very good (more than 1,600 m); ${ }^{\mathrm{c}} \mathrm{Grading}$ of magnetic resonance imaging (MRI): grade 3, severe stenosis; grade 2; moderate stenosis; grade 1, mild stenosis; and grade 0, no lumbar stenosis; ${ }^{\mathrm{d}}$ The stenosis ratio (SR) is the cross-sectional area of the canal at the axial MRI image with greatest neurologic compression at disc level over the

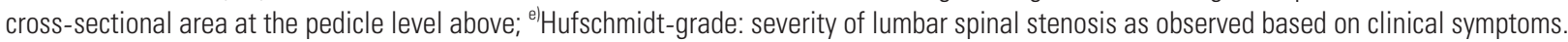

Table 3. Summary of CLSCS criteria and score ${ }^{\text {a) }}$

\begin{tabular}{lcccc} 
& \multicolumn{3}{c}{ Weights of 4 categorize } \\
\cline { 2 - 5 } & SPWT $^{\text {b) }}$ & Grading of MRI & SR $^{\text {d) }}$ & Hufschmidt-grade $^{\mathrm{e})}$ \\
No & 0.5 & 1 & 1 & 1.5 \\
Mild & 1 & 2 & 2 & 2.5 \\
Moderate & 2 & 3.5 & 3 & 4 \\
Severe & 2.5 & 4.5 & 4 & 5.5 \\
\hline
\end{tabular}

${ }^{a}$ The score index of classifying lumbar spinal canal stenosis (CLSCS) was obtained by summing up the points of the four variables: SR, Hufschmidtgrade, SPWT, and grading of magnetic resonance imaging (MRI). The CLSCS score was divided into 4 categories: CLSCS $<7$ (grade 0 ); $7 \leq C L S C S<10$

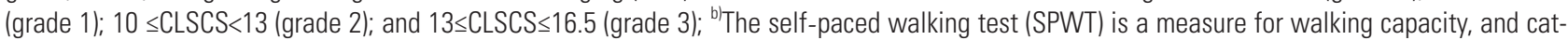
egorized as poor (less than $100 \mathrm{~m}$ ), fair (between $100 \mathrm{~m}$ and $800 \mathrm{~m}$ ), good (between $800 \mathrm{~m}$ and 1,600 m), and very good (more than 1,600 m); ${ }^{\mathrm{c}} \mathrm{Grad}$ -

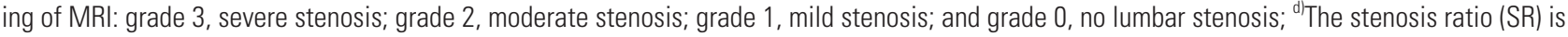
the cross-sectional area of the canal at the axial MRI image with greatest neurologic compression at disc level over the cross-sectional area at the

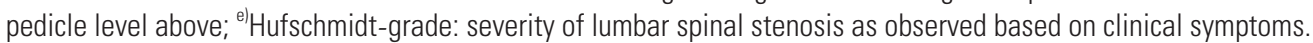


Table 4. Inter- and Intra-observer CLSCS grading system ( $\mathrm{n}=83$ pairs of repeat evaluations)

\begin{tabular}{lll} 
Variable & Intraobserver & Interobserver \\
Grade 0 & $0.77(0.68-0.81)$ & $0.79(0.69-0.81)$ \\
Grade 1 & $0.79(0.76-0.87)$ & $0.81(0.78-0.89)$ \\
Grade 2 & $0.84(0.81-0.89)$ & $0.88(0.82-0.92)$ \\
Grade 3 & $0.87(0.82-0.91)$ & $0.89(0.84-0.92)$ \\
CLSCS score & $0.82(0.81-0.93)$ & $0.84(0.82-0.96)$ \\
\hline
\end{tabular}

All values are expressed as kappa value (95\% confidence interval).

Interpretation of standard kappa: $<0.20$, poor agreement; $0.21-0.40$, fair agreement; $0.41-0.60$, moderate agreement; 0.61-0.80, good agreement; $0.81-1.00$, very good agreement, according to the interpretation of the kappa statistic by Landis and Koch [13].

CLSCS, classifying lumbar spinal canal stenosis.

Table 5. Treatment received within one years based on CLSCS grade ( $n=357)$

\begin{tabular}{lccc} 
& \multicolumn{2}{c}{ Treatment received within one years } & \\
\cline { 2 - 3 } CLSCS score & Surgery $(\mathrm{n}=171)$ & Non-surgery $(\mathrm{n}=186)$ & $<0.001$ \\
Grade 0 & 0 & 88 & $<0.001$ \\
Grade 1 & 9 & 94 & $<0.001$ \\
Grade 2 & 113 & 4 & $<0.001$ \\
Grade 3 & 49 & 0 & \\
\hline
\end{tabular}

The classifying lumbar spinal canal stenosis (CLSCS) score was divided into 4 categories: CLSCS $<7$ (grade 0); $7 \leq$ CLSCS $<10$ (grade 1); $10 \leq C L S C S<13$ (grade 2); and $13 \leq \mathrm{CLSCS} \leq 16.5$ (grade 3).

alDerived from T-test analysis.

published. Several real-world datasets for LSCS classification such as the Hufschmidt-grade, the morphology grade, and the grading of MRI have been presented in the literature $[10,11,14]$. The Hufschmidt-grade system is based on clinical symptoms and the other two systems are qualitative. Many studies have analyzed the relationship between dural sac cross-sectional area (DCSA) and the severity of symptoms in patients with LSCS, but its use for the classification of these LSCS patients remains controversial $[15,16]$. However, in an effort to improve the currently available classification criteria for LSCS, we designed a quantitative grading system using both subjective and objective parameters. It seems that the CLSCS score may be a useful tool for clinical decision making in LSCS patients. Finally, one should note that the CLSCS score in our study was developed and validated in the same population. Therefore, the authors recommend prospective testing of the CLSCS score in future trials for external validation to confirm its classification power.

Our own experience in clinical practice shows that many patients who received surgery had a low or mild grade on the four classification grading systems; the Hufschmidt-grade, the grading of MRI, the SPWT, and the SR; hence, it is expected that these patients should not have undergone surgery. Similarly, we saw many patients with a moderate or high grade on the four classification grading systems who did not receive surgery; however, they should have undergone surgery. In addition, the new system is better than the DCSA, morphology grade, and the grading of MRI, in the decision-making process due to the new tool; a higher CLSCS score correlated significantly with surgical intervention and a lower CLSCS score correlated significantly with conservative management. In addition, although the morphology grade and the grading of MRI are easy and reliable methods, we think that the new proposed system takes a step forward in preventing mistakes in practice and in assisting in the management of patients with borderline LSCS $[10,11,14]$.

There are a variety of instruments for assessing the performance or functionality in LSCS patients. These include 
the ODI, the NCOS, and the Swiss spinal stenosis questionnaire. However, there are no existing standard cut-off points for the definition of severity of LSCS for the data acquired by these instruments for the assessment of classification in these patients. If standard cut-off points are subsequently made available, such instruments may be useful in making accurate preoperative assessments.

There are limitations to this study. First, we were unable to identify the most effective classification parameter. However, multicenter studies are needed to prospectively examine these factors to establish a more accurate surgical indication assessment strategy. Perhaps the main weakness of this study is the fact that we did not use an external validation tool. The degree of stenosis on imaging and then correlation of the presenting symptoms and the CLSCS score with the outcome is important information for the readers. These findings should be confirmed in future studies in order to further enhance the clinical relevance of data. Finally, multicenter trials are necessary for further refinement of this scale.

\section{Conclusions}

The new index, the CLSCS score is generally reliable and valid for classifying patients with LSCS. Thus, the CLSCS score can be used in the decision-making process with respect to surgical intervention in these patients. Future multicenter clinical trials are needed to confirm these findings.

\section{Conflict of Interest}

No potential conflict of interest relevant to this article was reported.

\section{Acknowledgments}

The authors thank the staff of the Neurosurgery Unit at Imam-Hossain Hospital, Tehran, Iran.

\section{References}

1. Markman JD, Gaud KG. Lumbar spinal stenosis in older adults: current understanding and future directions. Clin Geriatr Med 2008;24:369-88.

2. de Schepper EI, Overdevest GM, Suri P, et al. Diagnosis of lumbar spinal stenosis: an updated system- atic review of the accuracy of diagnostic tests. Spine (Phila Pa 1976) 2013;38:E469-81.

3. Steurer J, Roner S, Gnannt R, Hodler J; LumbSten Research Collaboration. Quantitative radiologic criteria for the diagnosis of lumbar spinal stenosis: a systematic literature review. BMC Musculoskelet Disord 2011;12:175.

4. Laurencin CT, Lipson SJ, Senatus P, et al. The stenosis ratio: a new tool for the diagnosis of degenerative spinal stenosis. Int J Surg Investig 1999;1:127-31.

5. Hamanishi C, Matukura N, Fujita M, Tomihara M, Tanaka S. Cross-sectional area of the stenotic lumbar dural tube measured from the transverse views of magnetic resonance imaging. J Spinal Disord 1994;7: 388-93.

6. Tomkins CC, Battie MC, Rogers T, Jiang H, Petersen S. A criterion measure of walking capacity in lumbar spinal stenosis and its comparison with a treadmill protocol. Spine (Phila Pa 1976) 2009;34:2444-9.

7. Rainville J, Childs LA, Pena EB, et al. Quantification of walking ability in subjects with neurogenic claudication from lumbar spinal stenosis: a comparative study. Spine J 2012;12:101-9.

8. Tomkins-Lane CC, Battie MC. Validity and reproducibility of self-report measures of walking capacity in lumbar spinal stenosis. Spine (Phila Pa 1976) 2010; 35:2097-102.

9. Azimi P, Mohammadi HR, Montazeri A. An outcome measure of functionality in patients with lumber spinal stenosis: a validation study of the Iranian version of Neurogenic Claudication Outcome Score (NCOS). BMC Neurol 2012;12:101.

10. Lee GY, Lee JW, Choi HS, Oh KJ, Kang HS. A new grading system of lumbar central canal stenosis on MRI: an easy and reliable method. Skeletal Radiol 2011;40:1033-9.

11. Hufschmidt A, Bar M. Neurologie compact. Stuttgart: Thieme; 2006.

12. Mousavi SJ, Parnianpour M, Mehdian H, Montazeri A, Mobini B. The Oswestry Disability Index, the Roland-Morris Disability Questionnaire, and the Quebec Back Pain Disability Scale: translation and validation studies of the Iranian versions. Spine (Phila Pa 1976) 2006;31:E454-9.

13. Landis JR, Koch GG. The measurement of observer agreement for categorical data. Biometrics 1977;33: 159-74. 
14. Nunnally JC, Bernstein IH. Psychometric theory. 3rd ed. New York: McGraw-Hill; 1994.

15. Schizas C, Theumann N, Burn A, et al. Qualitative grading of severity of lumbar spinal stenosis based on the morphology of the dural sac on magnetic resonance images. Spine (Phila Pa 1976) 2010;35:191924.
16. Kanno H, Ozawa H, Koizumi Y, et al. Dynamic change of dural sac cross-sectional area in axial loaded magnetic resonance imaging correlates with the severity of clinical symptoms in patients with lumbar spinal canal stenosis. Spine (Phila Pa 1976) 2012;37:207-13. 